\title{
Análise das relações da curva de crescimento e eficiência produtiva de vacas da raça Holandesa ${ }^{1}$
}

\author{
Janaína Galvão Coelho², Pedro Franklin Barbosa ${ }^{3}$, Humberto Tonhatii ${ }^{4}$, Maria Armênia \\ Ramalho de Freitas ${ }^{5}$
}

\author{
1 Pesquisa financiada pela Embrapa Pecuária Sudeste, São Carlos, SP \\ 2 Mestranda em Genética e Melhoramento Animal. UNESP/FCAV, Jaboticabal, SP. \\ ${ }^{3}$ Embrapa Pecuária Sudeste - São Carlos, SP \\ ${ }^{4}$ Departamento de Zootecnia, FCAVIUNESP - Jaboticabal, SP \\ ${ }^{5}$ Associação Nacional de Criadores e Pesquisadores - Ribeirão Preto, SP.
}

RESUMO - O objetivo neste trabalho foi analisar as relações entre parâmetros da curva de crescimento (peso à maturidade e taxa de maturação) e medidas da eficiência produtiva de 333 vacas da raça Holandesa nascidas de 1992 a 2002 . Dados de idade ao primeiro parto, produção de leite e duração da primeira lactação, primeiro intervalo de partos e produção de leite por dia de intervalo de partos foram analisados por meio de um modelo linear generalizado com o efeito fixo de grupo contemporâneo (ano-estação de nascimento), os efeitos aleatórios de pai da vaca e erro e os efeitos lineares e quadráticos do peso à maturidade e da taxa de maturação. Nas análises de longevidade e de duração da vida útil, incluiu-se o efeito fixo de motivo de descarte da vaca. Houve efeitos linear e quadrático da taxa de maturação sobre a maioria das características, exceto o primeiro intervalo de partos e a produção de leite por dia de intervalo de partos. As estimativas das taxas de maturação ótimas variaram de 0,0896 a $0,1187 \mathrm{~kg} / \mathrm{kg}$ de peso vivo/mês. O peso à maturidade influenciou a longevidade de forma linear e quadrática. A combinação ótima foi de $701 \mathrm{~kg}$ de peso à maturidade e $0,0934 \mathrm{~kg} / \mathrm{kg}$ de peso vivo/mês de taxa de maturação. O peso à maturidade correlacionou-se de forma desfavorável com a duração da primeira lactação $(-0,11)$. Coeficientes de correlação significativos foram obtidos entre taxa de maturação e idade ao primeiro parto $(-0,22)$, produção de leite na primeira lactação $(0,18)$, duração da primeira lactação $(0,18)$ e longevidade $(0,14)$, o que confirma a hipótese de que vacas com taxas de maturação mais rápidas apresentam maior eficiência produtiva.

Palavras-chave: bovinos de leite, correlações, eficiência produtiva, peso à maturidade, taxa de maturação

\section{Analysis of the relationships of growth curve and productive efficiency of Holstein-Friesian cows}

\begin{abstract}
The objective of this study was to analyze the relationships between previously estimated individual growth curve parameters (mature weight and maturing rate) and measures of productive efficiency of 333 Holstein-Friesian cows, born from 1992 to 2002. Data on age at first calving, first lactation milk yield, first lactation length, first calving interval and milk yield per day of first calving interval were analyzed using a generalized linear model with the fixed effect for age group (year-season of birth), the random effects of sire and error and the linear and quadratic effects of mature weight and maturing rate. In the analyses of longevity and productive life, the fixed effect of culling reason was also included in the model. There were significant linear and quadratic effects of maturing rate on most of the traits analyzed, except on first calving interval and milk yield per day of first calving interval. The estimates of optimum maturing rates varied from 0.0896 to $0.1187 \mathrm{~kg} /$ $\mathrm{kg}$ of liveweight/month. There were both linear and quadratic effects of mature weight on longevity. The optimum combination was a mature weight of $701 \mathrm{~kg}$ and a maturing rate of $0.0934 \mathrm{~kg} / \mathrm{kg}$ of liveweight/month. Mature weight showed a significant and unfavourable correlation with first lactation length (-0.11). Significant correlation coefficients were obtained between maturing rate and age at first calving (-0.22), first lactation milk yield (0.18), first lactation length (0.18) and longevity (0.14), confirming the hypothesis that cows with faster maturing rates have higher productive efficiency.
\end{abstract}

Key Words: correlation, dairy cattle, mature weight, maturing rate, productive efficiency 


\section{Introdução}

Resultados de pesquisa obtidos no Brasil (Madalena, 1989; Madalena et al., 1990) têm mostrado que a eficiência produtiva dos rebanhos leiteiros pode ser melhorada com o uso de tecnologias apropriadas e de animais de potencial genético adequado à finalidade do sistema de produção. No melhoramento de bovinos de leite, tanto a menor idade ao primeiro parto quanto o grande número de ciclos reprodutivos no rebanho sem problemas são desejáveis. De acordo com Essl (1998), o problema é determinar como esses dois objetivos se combinam em termos biológicos.

A idade ao primeiro parto é um dos indicadores da eficiência produtiva. Em geral, fêmeas com menor idade ao primeiro parto apresentam maior vida produtiva (Strandberg, 1992). A longevidade (Hudson \& Van Vleck, 1981) tem grande importância econômica e as estimativas do valor econômico relativo dessa característica variam de 20 a $60 \%$ do valor econômico da produção de leite (Dekkers \& Jairath, 1994). Problemas de sobrevivência das raças leiteiras da espécie Bos taurus nos trópicos foram relatados por Vaccaro (1990).

Outro ponto importante é que a longevidade deveria ser considerada na estratégia de seleção. Embora com estimativa de herdabilidade baixa $(<0,10)$, na maioria dos trabalhos relatados na literatura (Bijma \& Jensen, 1996; Vollema \& Groen, 1996), estudos recentes (Martinez et al., 2005) têm revelado outras formas de avaliar a longevidade: por exemplo, habilidade de permanência até 48 meses é de herdabilidade mais alta $(>0,25)$.

Resultados de pesquisa relacionando os parâmetros da curva de crescimento com medidas de eficiência produtiva são escassos na literatura brasileira (Freitas et al., 1997). No Brasil, poucos trabalhos foram realizados sobre a duração da vida útil de vacas leiteiras. Lemos et al. (1996) estudaram a vida útil de 527 fêmeas de seis cruzamentos Holandês Vermelho-e-Branco (HVB) × Guzerá (1/4, 1/2, 5/8, $3 / 4,7 / 8$ e $\geq 31 / 32 \mathrm{HVB}$ ) e observaram que as vacas $\mathrm{F}_{1}$ HVB $\times$ Guzerá apresentaram menor taxa de descarte e duração da vida útil mais longa. Essas medidas de desempenho produtivo reduziram com o aumento da fração de HVB.

O objetivo neste trabalho foi analisar as relações entre os parâmetros da curva de crescimento e a eficiência produtiva de fêmeas da raça Holandesa Preta-e-Branca, criadas em um sistema de produção de leite na Região Sudeste do Brasil.

\section{Material e Métodos}

Os dados analisados neste trabalho foram obtidos das fichas individuais de registro zootécnico de 582 fêmeas da raça Holandesa nascidas no período de 1992 a 2002 e criadas no Sistema Intensivo de Produção de Leite do Centro de Pesquisa de Pecuária do Sudeste - Embrapa Pecuária Sudeste, São Carlos, São Paulo.

As estimativas dos parâmetros da curva de crescimento peso à maturidade e taxa de maturação foram obtidas pelo modelo não-linear de von Bertalanffy (1957), a partir de um arquivo de dados de 470 fêmeas com 11.685 pesagens do nascimento à idade adulta.

Para analisar as relações entre os parâmetros da curva de crescimento e as medidas de eficiência produtiva, os dados foram avaliados por meio do procedimento Box Plot do SAS (2000), eliminando-se as observações fora da curva da distribuição normal. Além disso, também foram eliminadas as informações dos touros com apenas uma filha no arquivo de dados e as fêmeas que não pariram. Assim, o número de observações variou de acordo com o grupo de características analisadas.

No Sistema Intensivo de Produção de Leite, as fêmeas eram mantidas em uma área de 96 hectares. Durante a época das águas (outubro a março), o regime alimentar era de pastagens dos capins tanzânia e tobiatã (Panicum maximum), elefante (Pennisetum purpureum Schum), andropogon (Andropogon gayanus) e braquiária (Brachiaria decumbens), manejadas em sistema de pastejo rotacionado. Durante a época da seca (abril a setembro), além das pastagens, a dieta das vacas era complementada com silagem de milho ou cana-de-açúcar com ureia. No decorrer do ano, independentemente da época, o rebanho recebeu suplementação com concentrados em quantidade e qualidade que variaram de acordo com a categoria animal (bezerras, novilhas, vacas secas e vacas em lactação).

As fêmeas foram inseminadas artificialmente com sêmen de touros da raça Holandesa provados para produção de leite. Os critérios de escolha dos touros foram as diferenças preditas para produção de leite, gordura e proteína, estatura, profundidade de úbere, ângulo de casco e facilidade de parto (especialmente para novilhas). Os bezerros machos foram descartados do rebanho logo após o nascimento e as bezerras, após a desmama, aos 60 dias de idade, foram criadas em lotes de acordo com a média do peso vivo individual e, depois, manejadas de acordo com seu desenvolvimento até atingirem idade e peso para entrada em reprodução (14-15 meses e 320-350 kg de peso 
vivo). O lote de inseminação de novilhas foi formado de acordo com esses critérios.

O manejo sanitário do rebanho (vacinações e outras medidas profiláticas) seguiu as práticas recomendadas para a região.

A análise dos dados de eficiência produtiva (idade ao primeiro parto, produção total de leite na primeira lactação, produção de leite em 305 dias de lactação, duração da primeira lactação, primeiro intervalo de partos, produção de leite por dia do primeiro intervalo de partos, longevidade e duração da vida útil) e o estudo da consistência da base de dados foram feitos utilizando-se os procedimentos disponíveis no Statistical Analysis System (SAS, 2000). Os dados de produção de leite na primeira lactação não foram corrigidos para a duração da lactação, seguindo recomendação de Madalena et al. (1992) de que não se deve corrigir a produção de leite para duração da lactação, tampouco projetar lactações para 305 dias.

Os grupos de contemporâneas foram formados pela combinação de ano e estação de nascimento (verão = janeiro a março; outono = abril a junho; inverno = julho a setembro; primavera $=$ outubro a dezembro) com pelo menos cinco observações. Os grupos com menos de cinco observações foram alocados ao grupo contemporâneo mais próximo da data de nascimento da maioria das fêmeas.

No estudo das relações entre as medidas de eficiência produtiva na primeira lactação (idade ao primeiro parto, produção total de leite, produção de leite até 305 dias de lactação, intervalo de partos, produção de leite por dia de intervalo de partos, duração da lactação, longevidade e duração da vida útil) e os parâmetros da curva de crescimento (peso à maturidade e taxa de maturação), os dados foram analisados pelo método dos quadrados mínimos ordinários utilizando-se o procedimento Generalized Linear Models (GLM) do Statistical Analysis System (SAS, 2000), pelo seguinte modelo linear geral:

$$
\begin{aligned}
Y_{i j k}= & \mu+G_{i}+b_{1}(A-\bar{A})+b_{2}(A-\bar{A})^{2}+b_{3}(k-\bar{k})+ \\
& b_{4}(k-\bar{k})^{2}+T_{j}+\varepsilon_{i j k}
\end{aligned}
$$

em que: $\mathrm{Y}_{\mathrm{ijk}}=$ valor observado na k-ésima vaca para cada caracterísitica independente (idade ao primeiro parto, produção total de leite, produção de leite até 305 dias de lactação, intervalo de partos, produção de leite por dia de intervalo de partos, duração da lactação, longevidade e duração da vida útil), pertencente ao i-ésimo grupo de contemporâneas, filha do j-ésimo touro; $\mu$ = média geral de cada característica de eficiência produtiva analisada; $\mathrm{GC}_{\mathrm{i}}=$ efeito fixo do i-ésimo grupo de contemporâneas; $\mathrm{b}_{1}$ = coeficiente linear de regressão do peso à maturidade; $\mathrm{A}=$ peso à maturidade do k-ésimo animal; $\overline{\mathrm{A}}=$ média do peso à maturidade; $\mathrm{b}_{2}=$ coeficiente quadrático de regressão do peso à maturidade; $\mathrm{k}$ = taxa de maturação do k-ésimo animal; $\overline{\mathrm{k}}=$ média da taxa de maturação; $\mathrm{b}_{3}=$ coeficiente linear de regressão da taxa de maturação; $\mathrm{b}_{4}=$ coeficiente quadrático de regressão da taxa de maturação; $T_{j}=$ efeito aleatório do j-ésimo touro (pai da vaca); e $\varepsilon_{i j k}=$ erro aleatório associado a cada valor observado.

Nas análises dos dados de longevidade e duração da vida útil, o efeito de causa de descarte (morte, venda para reprodução, venda para abate) foi incluído no modelo estatístico descrito anteriormente.

Os coeficientes de correlação de Pearson entre os parâmetros da curva de crescimento e as medidas de eficiência produtiva foram estimados utilizando-se o procedimento CORR do SAS (2000) com o objetivo de medir o grau de associação entre os valores fenotípicos e as características analisadas.

Tabela 1 - Frequências observadas para idade ao primeiro parto de vacas da raça Holandesa em cada grupo de contemporâneas (ano-estação

\begin{tabular}{|c|c|c|c|c|c|}
\hline Ano de nascimento & Verão & Outono & Inverno & Primavera & Total \\
\hline 1992 & 6 & 0 & 14 & 7 & 27 \\
\hline 1993 & 5 & 0 & 8 & 7 & 20 \\
\hline 1994 & 6 & 8 & 9 & 0 & 23 \\
\hline 1995 & 15 & 8 & 6 & 10 & 39 \\
\hline 1996 & 13 & 7 & 7 & 7 & 34 \\
\hline 1997 & 19 & 13 & 8 & 6 & 46 \\
\hline 1998 & 11 & 13 & 0 & 13 & 37 \\
\hline 1999 & 9 & 17 & 5 & 12 & 43 \\
\hline 2000 & 12 & 8 & 0 & 7 & 27 \\
\hline 2001 & 8 & 19 & 0 & 0 & 27 \\
\hline 2002 & 0 & 10 & 0 & 0 & 10 \\
\hline Total & 104 & 103 & 57 & 69 & 333 \\
\hline
\end{tabular}
de nascimento) - Sistema Intensivo de Produção de Leite, Embrapa Pecuária Sudeste, São Carlos, São Paulo 
As estações de nascimento (Tabela 1) foram classificadas em: verão $=$ janeiro a março; outono $=$ abril a junho; inverno = julho a setembro; e primavera = outubro a dezembro. O número de grupos de contemporâneas variou de 29 para duração da vida útil a 34 para idade ao primeiro parto.

\section{Resultados e Discussão}

As médias estimadas (erros-padrão) no rebanho para peso à maturidade e taxa de maturação obtidas por meio do uso do modelo não-linear de von Bertalanffy (1957) foram 661 (5) kg e $0,0794(0,0008) \mathrm{kg} / \mathrm{kg}$ de peso vivo/mês, respectivamente. No Brasil, Freitas et al. (1997), utilizando dados de 85 novilhas da raça Holandesa, pesadas todos os meses desde o nascimento até 24 meses de idade, e o modelo de Brody, obtiveram médias de 660,7 kg e 0,057 kg/ $\mathrm{kg} /$ mês para peso à maturidade e taxa de maturação, respectivamente. Na Espanha, Pérez-Cabal et al. (2006), utilizando o modelo não-linear de von Bertalanffy (1957), obtiveram estimativa do peso à maturidade de vacas da raça Holandesa de $647 \mathrm{~kg}$.

A média estimada da idade ao primeiro parto $(n=333)$ foi de 27,2 meses, semelhante à encontrada por Ribeiro et al. (2003), em rebanhos leiteiros do Estado de Kentucky nos Estados Unidos (28,0 meses), por Wolff et al. (2004), em rebanhos da raça Holandesa na bacia leiteira de Castrolanda, estado do Paraná (27,0 meses), por Val et al. (2004), também com média de 27,0 meses em um rebanho da raça Holandesa sob manejo intensivo no estado de São Paulo, e por Pérez-Cabal et al. (2006), em rebanhos da raça Holandesa na Espanha (28,2 meses). O resultado obtido é reflexo das boas práticas de alimentação e manejo reprodutivo das novilhas que são adotadas no sistema de produção de leite, como a entrada em reprodução aos 14-15 meses de idade com 320-350 kg de peso, o que não significa que todas as novilhas ficam prenhes nessa faixa de idade e, por isso, a média da idade ao primeiro parto foi de 27,2 meses.

No Brasil, em estudos realizados nas últimas duas décadas com a raça Holandesa em diversas bacias leiteiras do estado do Paraná (Basile et al., 1986; Rorato et al., 1992), foram verificados valores superiores de idade ao primeiro parto (média de 31,9 meses). Leite et al. (2001), com dados de vacas da raça Holandesa criadas em uma estação experimental de Bagé, Rio Grande do Sul, também encontraram média superior à deste trabalho (37,1 meses). Esses resultados sugerem que as práticas de manejo reprodutivo adotadas nos sistemas de produção quanto à idade e ao peso de entrada em reprodução das novilhas são diferentes entre os estudos.
Das causas de variação estudadas, a análise de variância mostrou efeitos significativos $(\mathrm{P}<0,05)$ linear e quadrático da taxa de maturação sobre a idade ao primeiro parto.

A equação de regressão da idade ao primeiro parto (IPP) em função da taxa de maturação $(\mathrm{k})$ foi: IPP = 1.502,11 9.189,29(k)+43.395,54( $\left(\mathrm{k}^{2}\right)$, que indica a existência de uma taxa de maturação que reduz a idade ao primeiro parto, como pode ser verificado pela segunda derivada. Essa taxa de maturação foi igual a $0,1059 \mathrm{~kg} / \mathrm{kg}$ de peso vivo/mês e indica que novilhas com maior taxa de maturação são mais precoces.

As médias estimadas (erros-padrão) da produção total de leite na primeira lactação e da produção de leite em 305 dias de lactação ( $n$ = 295) foram de 6.650 (91) kg e 6.231 (74) $\mathrm{kg}$, respectivamente. Para produção total de leite na primeira lactação, Val et al. (2004) observaram média de 8.026 kg em vacas manejadas em sistema intensivo no estado de São Paulo e submetidas a três ordenhas diárias. Para produção de leite em 305 dias de lactação, Magalhães et al. (2006) e Vargas et al. (2006), trabalhando com dados de rebanhos da raça Holandesa do estado de São Paulo participantes do Programa de Análises de Rebanhos Leiteiros e da Qualidade do Leite, coordenado pela Escola Superior de Agricultura Luiz de Queiroz da Universidade de São Paulo, relataram médias de 6.640 e $6.634 \mathrm{~kg}$, respectivamente. Melo et al.(2005) relataram média de produção de leite em 305 dias de lactação de primíparas da raça Holandesa criadas nos estados de Minas Gerais, São Paulo, Rio Grande do Sul, Santa Catarina e Espírito Santo de $6.246 \mathrm{~kg}$. Esses resultados, semelhantes aos observados neste trabalho, comprovam o potencial de produção de vacas primíparas da raça Holandesa criadas em sistemas de produção com boas práticas de manejo e alimentação.

A média estimada (erro-padrão) da duração da primeira lactação foi igual a 315,5 (3,2) dias, inferior à observada por Val et al. (2004) em rebanho da raça Holandesa sob manejo intensivo, com três ordenhas diárias, no estado de São Paulo (346,4 dias), mas dentro do período de lactação de 305 dias recomendado para vacas leiteiras (Schmidt et al., 1988). Melo et al. (2005) observaram média de duração da primeira lactação de 291 dias em primíparas criadas em 531 rebanhos de diversos estados das regiões Sudeste e Sul do Brasil.

Para as produções de leite, houve efeitos significativos apenas da regressão linear da taxa de maturação. As equações de regressão obtidas para produção total de leite na primeira lactação (PL) e produção de leite em 305 dias de lactação (P305) em função da taxa de maturação (k) foram:

$$
\begin{aligned}
& \mathrm{PL}=-2.935,34+261.266(\mathrm{k})-1.127 .649,37\left(\mathrm{k}^{2}\right) \\
& \mathrm{P} 305=-2.516,10+192.650,33(\mathrm{k})-811.515,82\left(\mathrm{k}^{2}\right)
\end{aligned}
$$

As taxas de maturação que aumentam as produções de leite foram iguais a 0,1158 e $0,1187 \mathrm{~kg} / \mathrm{kg}$ de peso vivo/mês 
para PL e P305 respectivamente, como pode ser verificado pela derivada segunda de cada equação.

Para a duração da primeira lactação (DL; n = 295), houve efeitos significativos linear e quadrático da taxa de maturação (k), o que indica a existência de uma taxa de maturação (k) que otimiza a duração da primeira lactação. A equação de regressão obtida foi:

$$
\mathrm{DL}=-38,90+9.612,28(\mathrm{k})-48.040,03\left(\mathrm{k}^{2}\right)
$$

A taxa de maturação que aumenta a duração da primeira lactação foi de $0,10 \mathrm{~kg} / \mathrm{kg}$ de peso vivo/mês. Na literatura não foram encontrados outros trabalhos relatando esse tipo de relação. Os resultados obtidos indicam que, em vacas com taxas de maturação maiores ou menores que $0,10 \mathrm{~kg} / \mathrm{kg}$ de peso vivo/mês, as primeiras lactações são mais curtas, o que sugere a existência de um ponto de equilíbrio entre essas características.

A média estimada (erro-padrão) do primeiro intervalo de partos ( $\mathrm{n}=214)$ foi de 391,2 (4,2) dias, com coeficiente de variação de $15,69 \%$. Esse resultado é superior aos relatados na literatura brasileira para a raça Holandesa e reflete o manejo diferenciado adotado no sistema de produção de leite da Embrapa Pecuária Sudeste para as vacas primíparas, que são manejadas em lote único e alimentadas de acordo com suas exigências nutricionais, mas, mesmo assim, o primeiro intervalo de partos foi maior que o recomendado por Schmidt et al. (1988), de 365 dias. Madalena et al. (1983) obtiveram média estimada (erro-padrão) de 433 (23) dias para vacas da raça Holandesa criadas em um rebanho com alto nível de manejo (média da primeira lactação de 3.500 kg de leite) na Região Sul de Minas Gerais.

A média estimada (erro-padrão) da produção de leite por dia de intervalo de partos das primíparas $(n=214)$ foi igual a $17,17(0,26) \mathrm{kg}$, com coeficiente de variação de 22,02\%. A média obtida é superior àquelas relatadas para vacas da raça Holandesa classificadas em três ordens de parto, de $9,9(0,8) \mathrm{kg}$ (Madalena et al., 1983) e, para primíparas, de 7,01 $(0,45) \mathrm{kg}$ (Madalena et al., 1990). Esses resultados indicam que o nível de manejo alimentar das primíparas no sistema de produção de leite em estudo pode ser considerado alto.

Não houve efeito das causas de variação incluídas no modelo no primeiro intervalo de partos nem na produção de leite por dia de intervalo de partos. Hansen et al. (1999) e Pérez-Cabal et al. (2006) relataram que vacas da raça Holandesa mais pesadas à maturidade apresentaram primeiros intervalos de parto mais longos, o que não foi observado neste trabalho, provavelmente em razão das boas práticas de manejo das primíparas no sistema de produção de leite.

A média estimada (erro-padrão) da longevidade $(\mathrm{n}=256)$ foi de 55,64 $(0,99)$ meses, semelhante aos resultados simulados por Cardoso et al. (1999) no Brasil para animais da raça Holandesa (54,9 meses) e aos encontrados por Ribeiro et al. (2003) em rebanhos leiteiros do Kentucky, nos Estados Unidos (53,6 meses). No entanto, a média da longevidade foi inferior àquelas encontradas por Silva et al. (1986) em sete rebanhos das raças Guernsey (68,1 meses), Holandesa (67,9 meses) e Jersey (72,8 meses) no estado da Flórida, nos Estados Unidos.

Houve efeitos significativos (linear e quadrático) do peso à maturidade sobre a longevidade, portanto, existe um peso à maturidade que estende a longevidade. A equação de regressão da longevidade (LONG) em relação ao peso à maturidade foi:

$$
\text { LONG }=-8.251,25+17,9436(\mathrm{~A})-0,0128\left(\mathrm{~A}^{2}\right)
$$

Pela segunda derivada da equação de regressão, o peso ótimo à maturidade foi de $701 \mathrm{~kg}$. Hansen et al. (1999), em vacas da raça Holandesa selecionadas para menor e maior tamanho à maturidade, obtiveram médias de peso adulto de $641 \mathrm{~kg}$ e $720 \mathrm{~kg}$, respectivamente, e concluíram que a longevidade das vacas de tamanho menor foi $15,4 \%$ maior que a das vacas de tamanho maior.

Os valores que maximizam a longevidade são maiores que as médias observadas no rebanho para as fêmeas nascidas de 1992 a 2002 ( $\mathrm{A}=661 \mathrm{~kg} ; \mathrm{k}=0,0794 \mathrm{~kg} / \mathrm{kg}$ de peso vivo/mês, como já relatado anteriormente), sugerindo que há espaço para alterar, na direção desejada, os parâmetros da curva de crescimento, com maior amplitude para a taxa de maturação $(17,5 \%)$ que para o peso à maturidade (6,1\%). O problema é encontrar o ponto de equilíbrio entre esses parâmetros em termos biológicos, principalmente por causa da correlação negativa entre eles $(-0,80, \mathrm{P}<0,01)$, como obtido neste trabalho (Tabela 2$)$. Na literatura consultada não foram encontrados outros

Tabela 2 - Coeficientes de correlação de Pearson entre parâmetros da curva de crescimento e medidas de eficiência produtiva de vacas da raça Holandesa (Sistema Intensivo de Produção de Leite, Embrapa Pecuária Sudeste, São Carlos, São Paulo)

\begin{tabular}{lcc}
\hline Característica & $\begin{array}{c}\text { Peso à } \\
\text { maturidade }\end{array}$ & $\begin{array}{c}\text { Taxa de } \\
\text { maturação }\end{array}$ \\
\hline Peso à maturidade & 1,00 & $-0,80^{* *}$ \\
Taxa de maturação & $-0,80^{*}$ & 1,00 \\
Idade ao primeiro parto & 0,05 & $-0,22^{* *}$ \\
Produção de leite & $-0,05$ & $0,18^{* *}$ \\
Produção de leite até 305 dias & $-0,03$ & $0,17 * *$ \\
Duração da primeira lactação & $-0,11^{*}$ & $0,18^{* *}$ \\
Primeiro intervalo de partos & 0,05 & 0,01 \\
Produção de leite/dia de intervalo & 0,04 & 0,06 \\
Longevidade & $-0,02$ & $0,14 *$ \\
Duração da vida útil & 0,06 & 0,04 \\
\hline
\end{tabular}

** $\mathrm{P}<0,01 ; * \mathrm{P}<0,05$ 
trabalhos abordando qual seria o ponto de equilíbrio entre os parâmetros da curva de crescimento de vacas da raça Holandesa.

A ausência de efeito significativo de grupo contemporâneo na longevidade indica que as condições climáticas, as práticas de criação e manejo e as políticas de descarte tiveram efeitos semelhantes na longevidade das vacas ao longo do período estudado.

O mesmo pode ser dito com relação ao motivo de descarte. A maioria (52,34\%) das fêmeas foi descartada para abate devido a falhas reprodutivas, problemas de patas e pernas, acidentes, doenças e idade avançada (descarte involuntário); 28,52\% das fêmeas foram descartadas e vendidas para reprodução por apresentar eficiência produtiva abaixo da média do rebanho (descarte voluntário) e $19,14 \%$ foram descartadas por morte (descarte involuntário). Na Região Sudeste, Lemos et al. (1996) relataram taxas de descarte de vacas da raça Holandesa, criadas em fazendas com alto nível de manejo, de $41,2 \%$ por morte, $52,9 \%$ por venda para abate e apenas $5,9 \%$ por venda para produção de leite, contrastando com as taxas de descarte por venda para produção de leite e por morte obtidas neste trabalho, mas deve ser ressaltado que, por motivos contratuais entre a Embrapa Gado de Leite e os produtores, as vacas podiam ser descartadas do rebanho por qualquer razão, exceto por baixa produção de leite. A taxa de descarte por morte foi menor no sistema de produção de leite da Embrapa Pecuária Sudeste que aquela observada por Lemos et al. (1996) em rebanhos com alto nível de manejo, provavelmente devido às boas práticas de manejo sanitário adotadas.

Essl (1998) recomendou uma estratégia de melhoramento para aumentar o lucro durante a vida produtiva por meio da redução das causas de descarte involuntário, em vez do melhoramento da longevidade propriamente dita. Considerando que a maioria das fêmeas neste estudo (71,5\%) foi descartada do rebanho de maneira involuntária, essa estratégia parece ser interessante, a não ser que melhores indicadores da longevidade sejam encontrados no futuro.

A média estimada (erro-padrão) da duração da vida útil das vacas $(\mathrm{n}=224)$ foi de 31,10 $(0,89)$ meses, muito menor que a relatada por Lemos et al. (1996) para vacas puras por cruza da raça Holandesa Vermelha e Branca criadas em nível alto de manejo (82,8 meses) participantes de um projeto de pesquisa sobre avaliação de estratégias de cruzamento entre raças leiteiras na Região Sudeste, que podiam ser descartadas por qualquer razão, exceto por baixa produção. Por este motivo, segundo Lemos et al. (1996), os produtores foram menos rigorosos no descarte das vacas de menor eficiência, o que aumentou consideravelmente a vida útil.
A média da duração da vida útil das vacas obtida neste trabalho foi semelhante àquelas encontradas por Silva et al. (1986) em rebanhos da raça Holandesa na Flórida, Estados Unidos (34,8 meses), Nilforooshan \& Edriss (2004) com dados de 45 rebanhos da raça Holandesa criados na Província de Isfahan, Irã (30,1 meses), e superior à encontrada por Ribeiro et al. (2003) em rebanhos leiteiros do estado de Kentucky nos Estados Unidos (25,6 meses).

Houve efeitos significativos de grupo de contemporâneas $(\mathrm{P}<0,05)$ e da taxa de maturação (linear e quadrático, $\mathrm{P}<0,01)$ na duração da vida útil das vacas.

O efeito significativo de grupo de contemporâneas foi devido aos descartes de grande parte das fêmeas nascidas em 1992 e em 1997, por baixa produção de leite na primeira lactação, e ao fato de a maioria das fêmeas nascidas em 2000 e 2001 ainda estarem no rebanho em dezembro de 2004, data-limite considerada para o cálculo da duração da vida útil.

A equação de regressão da duração da vida útil (DVU) em função da taxa de maturação $(\mathrm{k})$ foi a seguinte:

DVU $=-5.609,42+97.709,70(k)-545.064,38\left(k^{2}\right)$

Isso resulta em uma taxa de maturação que aumenta a duração da vida útil para $0,0896 \mathrm{~kg} / \mathrm{kg}$ de peso vivo/mês, como pode ser verificado pelo sinal da derivada segunda da equação de regressão.

O coeficiente de correlação entre o peso à maturidade e a taxa de maturação (Tabela 2) foi igual a $-0,80(\mathrm{P}<0,01)$ e indica que, quanto maior a taxa de maturação, menor o peso à maturidade e vice-versa. Em fêmeas da raça Holandesa criadas no Brasil, Freitas et al. (1997) encontraram coeficiente de correlação de -0,53 entre esses parâmetros.

Com relação ao peso à maturidade e às medidas de eficiência produtiva, apenas o coeficiente de correlação com a duração da primeira lactação foi significativo $(\mathrm{P}<0,05)$ e na direção desfavorável $(-0,11)$, ou seja, as vacas com maior peso à maturidade apresentaram menor duração da primeira lactação, embora o coeficiente de correlação tenha sido baixo. Esse resultado é diferente do relatado por Bayram et al. (2004), que encontraram correlação positiva $(r=0,25)$ entre o peso à maturidade e a duração da lactação de vacas da raça Pardo-Suíça.

A taxa de maturação correlacionou-se significativamente ( $\mathrm{P}<0,01)$ com a idade ao primeiro parto $(-0,22)$, a produção de leite $(0,18)$, a produção de leite em 305 dias $(0,17)$ e a duração da primeira lactação $(0,18)$. Esses resultados sugerem que vacas com maiores taxas de maturação apresentam melhor eficiência produtiva, quando consideradas as características medidas na primeira lactação. Na literatura não foram encontrados outros trabalhos relatando esse tipo de resultado. 


\section{Conclusões}

Há valores da taxa de maturação, como parâmetro da curva de crescimento de bovinos, que melhoram parâmetros como a idade ao primeiro parto, a produção de leite na primeira lactação, a duração da primeira lactação e a duração da vida útil de vacas da raça Holandesa. Da mesma forma, há também um peso à maturidade que aumenta a longevidade de vacas da raça Holandesa criadas em sistemas intensivos de produção de leite na Região Sudeste do Brasil. Embora com coeficientes de baixa magnitude, a taxa de maturação correlaciona-se de forma favorável com a idade ao primeiro parto, a produção de leite na primeira lactação, a duração da primeira lactação e a longevidade de vacas da raça Holandesa, o que confirma a hipótese de que vacas de maior peso à maturidade apresentam menor eficiência produtiva quanto às características observadas na primeira lactação.

\section{Literatura Citada}

BASILE, J.R.; RIBAS, N.P.; KOEHLER, H.S. Efeito de meio ambiente na idade ao primeiro parto de um rebanho Holandês (malhado de preto e malhado de vermelho) criado no Estado do Paraná. Revista Brasileira de Reprodução Animal, v.10, n.3, p.137-145, 1986.

BAYRAM, B.; YANAR, M.; AKBULUT, O. Relationships among Richards growth curve parameters, reproductive and milk production traits in Brown Swiss cattle. Journal of Applied Animal Research, v.26, n.1, p.29-32, 2004.

BIJMA, P.; JENSEN, J. Genetic analysis of herd life and stayability in Danish dairy cattle. In: INTERNATIONAL WORKSHOP ON GENETIC IMPROVEMENT OF FUNCTIONAL TRAITS IN CATTLE, Genbloux, Belgium. Proceedings... Interbull, Uppsala, Sweden: 1996. p.150-156.

CARDOSO, V.L.; NOGUEIRA, J.R.; Van ARENDONK, J.A.M. Optimum replacement and insemination policies for Holstein cattle in the southeastern region of Brazil: The effect of selling animals for production. Journal of Dairy Science, v.82, p.1449-1458, 1999.

DEKKERS, J.C.M.; JAIRATH, L.K. Requirements and uses of genetic evaluations for conformation and herd life. In: WORLD CONGRESS OF GENETICS APPLIED TO LIVESTOCK PRODUCTION, 5., 1994, Guelph. Proceedings... Guelph, 1994. p.61-68.

ESSL, A. Longevity in dairy cattle breeding: a review. Livestock Production Science, v.57, p.79-89, 1998.

FREITAS, A.F.; DURÃES, M.C.; TEIXEIRA, N.M. Curvas de crescimento de novilhas da raça Holandesa mantidas em regime de confinamento. Arquivo Brasileiro de Medicina Veterinária e Zootecnia, v.49, n.1, p.85-93, 1997.

HANSEN, L.B.; COLE, J.B.; MARX, G.D. et al. Productive life and reasons for disposal of Holstein cows selected for large versus small body size. Journal of Dairy Science, v.82, n.4, p.795-801, 1999.

HUDSON, G.F.S.; VAN VLECK, L.D. Relationship between production and stayability in Holstein cattle. Journal of Dairy Science, v.64, p.2246-2250, 1981.

LEMOS, A.M.; TEODORO, R.L.; MADALENA, F.E. Comparative performance of six Holstein-Friesian x Guzera grades in Brazil. 9. Stayability, herd life and reason for disposal.
Brazilian Journal of Genetics, v.19, n.2, p.259-264, 1996.

LEITE, T.E.; MORAES, J.C.F.; PIMENTEL, C.A. Eficiência produtiva e reprodutiva em vacas leiteiras. Ciência Rural, v.31, n.3, p.467-472, 2001.

MADALENA, F.E. Cattle breed resource utilization for dairy production in Brazil. Brazilian Journal of Genetics, v.2, n.3, p.183-220, 1989 (suppl.).

MADALENA, F.E.; VALENTE, J.; TEODORO, R.L. et al. Produção de leite e intervalo de partos de vacas HPB e mestiças HPB:Gir num alto nível de manejo. Pesquisa Agropecuária Brasileira, v.18, n.2, p.195-200, 1983.

MADALENA, F.E.; LEMOS, A.M.; TEODORO, R.L. et al. Dairy production and reproduction in Holstein-Friesian and Guzera crosses. Journal of Dairy Science, v.73, n.7, p.1872-1886, 1990.

MADALENA, F.E.; LEMOS, A.M.; TEODORO, R.L. Consequences of removing the variation in lactation length on the evaluation of dairy cattle breeds and crosses. Brazilian Journal of Genetics, v.15, n.3, p.585-594, 1992.

MAGALHÃES, H.R.; EL FARO, L.; CARDOSO, V.L. et al Influência de fatores de ambiente sobre a contagem de células somáticas e sua relação com perdas na produção de leite de vacas da raça Holandesa. Revista Brasileira de Zootecnia, v.35, n.2, p.415-421, 2006.

MARTINEZ, G.E.; KOCH, R.M.; CUNDIFF, L.V. et al. Genetic parameters for stayability, stayabaility at calving and stayabaility at weaning to specified ages for Hereford cows Journal of Animal Science, v.83, p.2033-2042, 2005.

MELO, C.M.R.; PACKER, I.U.; COSTA, C.N. et al. Parâmetros genéticos para as produções de leite no dia do controle e da primeira lactação de vacas da raça Holandesa. Revista Brasileira de Zootecnia, v.34, n.3, p.796-806, 2005.

NILFOROOSHAN, M.A.; EDRISS, M.A. Effect of age at first calving on some productive and longevity traits in Iranian Holsteins of the Isfahan Province. Journal of Dairy Science, v.87, n.7, p.2130-2135, 2004.

PÉREZ-CABAL， M.A.; GONZÁLEZ SANTILLANA, R.; ALENDA, R. Mature body weight and profit selection in Spanish dairy cattle. Livestock Science, v.99, n.2-3, p.257-266, 2006.

RIBEIRO, A.C.; MCALLISTER, A.J; QUEIROZ, S.A. Efeitos das taxas de descarte sobre medidas econômicas de vacas leiteiras em Kentucky. Revista Brasileira de Zootecnia, v.32, n.6, p.1737-1746, 2003 (supl. 1).

RORATO, P.R.N.; RIBAS, N.P.; LÔBO, R.B. et al. Interação genótipo-ambiente no desempenho produtivo de vacas da raça Holandesa no estado do Paraná. Revista da Sociedade Brasileira de Zootecnia, v.21, n.1, p.57-67, 1992.

SCHMIDT, G.H.; VAN VLECK, L.D.; HUTJENS, M.F. Principles of dairy science. Prentice Hall, 1988. 466p.

SILVA, H.M.; WILCOX, C.J.; SPURLOCK, A.H. et al. Factors affecting age at first parturition, life span, and vital statistics of Florida dairy cows. Journal of Dairy Science, v.69, n.2, p.470-476, 1986.

STATISTICAL ANALYSIS SYSTEM - SAS. Stat user's guide. Cary: SAS Institute, 2000. (CD-ROM).

STRANDBERG, E. Lifetime performance in dairy cattle. Acta Agriculturae Scandinavica, v.42, p.71-81, 1992.

VACCARO, L.P. Survival of European dairy breeds and their crosses with Zebu in the Tropics. Animal Breeding Abstracts, v.58, p.475-494, 1990.

VAL, J.E.; FREITAS, M.A.R.; OLIVEIRA, H.N. et al. Indicadores de desempenho em rebanho da raça Holandesa: curvas de crescimento e altura, características reprodutivas, produtivas e parâmetros genéticos. Arquivo Brasileiro de Medicina Veterinária e Zootecnia, v.56, n.1, p.86-93, 2004 . 
VARGAS, A.D.F.; EL FARO, L.; CARDOSO, V.L. et al. Estimação de parâmetros genéticos para a produção de leite no dia do controle e em 305 dias para primeiras lactações de vacas da raça Holandesa. Revista Brasileira de Zootecnia, v.35, n.5, p.1959-1965, 2006.

VOLLEMA, A.R.; GROEN, R.F. Genetic parameters of longevity traits of an upgrading population of dairy cattle. Journal of Dairy Science, v.79, n.12, p.2261-2267, 1996.
Von BERTALANFFY, L. Quantitative laws in metabolism and growth. The Quartely Review of Biology, v.32, p.217-230, 1957.

WOLFF, M.C.C.; MONARDES, H.G.; RIBAS, N.P. Fatores ambientais sobre a idade ao primeiro parto, dias abertos e intervalo entre partos em vacas da raça Holandesa na bacia leiteira de Castrolanda, estado do Paraná. Archives of Veterinary Science, v.9, n.2, p.35-41, 2004. 